\title{
INFLUENCE OF POROSITY AND CELL DENSITY ON TISSUE ENGINEERING OF MANDIBULAR CONDYLAR CARTILAGE
}

\author{
S. Cortez (1), A. Completo (2), J.L. Alves (1) \\ 1. CMEMS - University of Minho, scortez@dem.uminho.pt; \\ 2. TEMA - University of Aveiro, completo@ua.pt;
}

\begin{abstract}
Introduction
The condylar cartilage plays a crucial role in the normal movement of the temporomandibular joint. When the degeneration in this tissue occurs, this joint loses functionality and one possible solution for healing is the implantable materials produced by tissue engineering [1]. The development of artificial cartilage by this technique depends largely on the supply of a suitable amount of nutrients, including oxygen and glucose, to the chondrocytes during the culture process within the bioreactor [2].

The concentration of cells within the scaffold and the porosity of scaffold can affect the diffusion of nutrients essential to cell proliferation.

Through a numerical approach, the aim of this work is to understand how the cellular density and porosity in the scaffold can affect the engineered cartilage growth and compromise its mechanical properties.
\end{abstract}

\section{Methodology}

In this work, a mathematical formulation to analyze the nutrient diffusion, the cell growth as well as the solid matrix evolution of the tissue-engineered condylar cartilage is presented.

Partial differential equations were modelled to predict the nutrients, cells, glycosaminoglycans and collagen inside the scaffold. The transport of nutrients in the scaffold was governed by the reaction-diffusion equation [3], including a diffusion coefficient, which depends on the solid fraction. The cell proliferation was modelled by the Contois kinetics, which includes the effect of glucose concentration [4]. To predict the solid matrix remodeling, a new growth model was implemented, based on the concentration of extracellular matrix components inside the construct. Numerical simulations were preformed using a 3D mesh of one-quarter of an agarose disc, considering different initial porosities and cell seeding densities. The simulation were performed during a static culture time of 1 week. The gradient profiles of the nutrients concentrations, distribution of cells and matrix constituents inside the scaffold were analyzed.

\section{Results and Discussion}

The results of the mathematical analysis revealed that porosity and cell seeding density in the scaffold influences the permeability of the structure, reducing the diffusion of nutrients and, consequently, reducing the cell proliferation. With an high intial cell density and a lower porosity, the supply of nutrients is compromised, leading to an heterogeneity of mechanical properties of the artificial tissue.

\section{Conclusion}

This model has proven useful to predict which scaffold's porosity and initial cells density would promote a good tissue growth. This simple mathematical model was developed to expect the experimental data, mimetizing numerically the tissue growth events in the production of engineered condylar cartilage.

\section{References}

[1] Wang L. et al, Osteoarthritis and Cartilage, 17(3): 346-353, 2009.

[2] Saini S., et al, Biotechnology progress, 19(2): 510-521, 2003.

[3] Lin T., et al, Biotechnology progress, 29(2):452462, 2013.

[4] Chung C., et al, Annals of biomedical engineering, 38(4):1655-1663, 2010.

\section{Acknowledgements}

The first author is grateful to FCT (Fundação para a Ciência $e$ Tecnologia) for the $\mathrm{PhD}$ grant (SFRH/BD/87933/2012). This work is supported by FCT with the reference projects UID/EEA/04436/2013, PEstC/EME/UI0481/2013, FCOMP-01-0124-FEDER015191, and by FEDER funds through the COMPETE 2020 - Programa Operacional Competitividade $e$ Internacionalização (POCI) with the reference projects POCI-01-0145-FEDER-006941 and COMPETE 2020 PTDC/ EMSTEC/ 3263/ 2014. 